\title{
The Power of Tensor-Based Approaches in Cardiac Applications
}

\author{
Sibasankar Padhy, Griet Goovaerts, Martijn Boussé, Lieven De Lathauwer, \\ and Sabine Van Huffel
}

\begin{abstract}
The electrocardiogram (ECG) is a biomedical signal that is widely used to monitor the heart and diagnose cardiac problems. Depending on the clinical need, the ECG is recorded with one or multiple leads (or channels) from different body locations. The signals from different ECG leads represent the cardiac activity in different spatial directions and are thus complementary to each other. In traditional methods, the ECG signal is represented as a vector or a matrix and processed to analyze temporal information. When multiple leads are present, most methods process each lead individually and combine decisions from all leads in a later stage. While this approach is popular, it fails to exploit the structural information captured by the different leads.

Recently, there is a trend towards the use of tensor-based methods in biomedical signal processing. These methods represent the signals by tensors, which are higher-order generalizations of vectors and matrices that allow the analysis of multiple modes simultaneously. In the past years, tensor decomposition methods have been applied on ECG signals to solve different clinical challenges. This chapter discusses the power of different tensor decompositions with a focus on typical ECG problems that can be solved using tensors.
\end{abstract}

The first two authors have equal contribution for the work.

S. Padhy, G. Goovaerts, M. Boussé, L. De Lathauwer and S. Van Huffel

Department of Electrical Engineering (ESAT), STADIUS Center for Dynamical Systems, Signal Processing and Data Analytics, KU Leuven, Leuven, Belgium.

e-mail: $\quad$ \{sibasankar.padhy, griet.goovaerts, martijn. bousse, lieven. delathauwer, sabine.vanhuffel\}@kuleuven. be

S. Padhy, G. Goovaerts and S. Van Huffel

imec, Leuven, Belgium.

L. De Lathauwer

Group Science, Engineering and Technology, KU Leuven Kulak, Kortrijk, Belgium. 


\section{Introduction}

The electrocardiogram (ECG) is a well-known diagnostic tool and one of the most preferred tests in every day clinical practice. It is widely-used in both hospitals and ambulatory environments because it is easy to measure and contains a large amount of information about the condition of the heart. Moreover, its associated cost is relatively low compared to imaging techniques such as echocardiography or magnetic resonance imaging.

In recent years, advances in sensor techniques and the introduction of wireless technologies have led to the development of various new ECG technologies, including wearable devices and smart phone set-ups. The rise of these novel technologies has introduced both opportunities and challenges in the field of ECG monitoring. Improvements in digital filters led to more accurate noise removal methods and increased signal qualities, which allow the detection and analysis of more refined ECG characteristics. Expansions of computing power and storage capacity permit the use of more advanced signal processing techniques and advances in material sciences have lead to the development of sensors that can be worn for many days in a row. Manual analysis of these large-scale ECG data sets has become tedious, time-consuming and expensive, leveraging the need for automated ECG processing methods that can analyze the data in a computationally efficient way.

In a clinical context, ECG signals are mostly recorded with different channels or leads, where each lead corresponds to the cardiac electrical signal viewed from a different spatial angle. The combination of these channels gives a global view from the heart in three dimensions. While matrices could technically be used to analyze these multidimensional signals by constructing multiple matrices for each spatial angle and concatenating them in one big matrix; there is no reason why the original multidimensionality of the data should not be preserved and exploited maximally. This way the information that is shared over all dimensions can be analyzed simultaneously. This can be done in a straightforward way through the use of tensors. Mathematically speaking, tensors are higher-order generalizations of vectors and matrices. Tensors tools have been applied extensively in various applications within signal processing, data mining, and machine learning [17, 45, 6, 75]. In biomedical signal processing, they have gained popularity in neuroscience applications such as epilepsy monitoring, brain tissue segmentation and EEG processing [7, 8, 20, 84].

The purpose of this chapter is to give an overview of different applications in ECG processing where tensors have been successfully used to process multilead ECG signals. Since tensors are a rather novel concept in ECG processing, the next section first gives a motivation for the use of tensor methods in ECG processing. In Section 3, the main concepts and methods related to tensors are summarized. We encourage interested readers to refer to the overview articles and references therein for a more comprehensive overview of tensor methods and their applications [17, 45, 6, 75]. Section 4 discusses the use 
of tensor methods in five specific ECG applications, where we highlight the flexible way tensors can be used to deal with specific ECG characteristics. Here, we discuss the tensor-based approaches to the third-order tensor. Finally, Section 5 concludes the chapter by suggesting possible directions for future research. The work described in this chapter, especially Sections 2 and 3 and the CPD-based methods, is largely based on [34].

\section{Motivation}

ECG signals can be measured with one or more leads, but multilead recordings are considered as the gold standard, especially in clinical contexts. Also, long-term recordings are often performed using Holter monitors which typically have a minimum of two channels. Each lead records the cardiac electrical activity from a different spatial angle, complementing each other and enabling a comprehensive three-dimensional view of the electrical activity of the heart.

Most methods that deal with multilead ECG data employ a late-integration approach, which means that they process each lead separately and afterwards combine the results of all channels [87, 47, 37]. This obviously fails to exploit the correlations between the different spatial angles, leading to a loss of available information. Tensor methods allow one to simultaneously analyze all channels, enabling exploitation of all the available information at once in contrast to late-integration methods. Hence, a tensor-based approach is clearly advantageous for applications which require the assessment of the global behaviour of the heart.

While matrix-based decomposition methods have been used successfully in many applications, they often put constraints on the signals in order to obtain unique and thus interpretable solutions. For example, Principal Component Analysis (PCA) requires the different components to be orthogonal to each other and Independent Component Analysis enforces independence between the different components. These assumptions are however not necessarily met in real-life conditions. However, in contrast to low-rank matrix models, lowrank tensor models are unique under mild conditions.

Finally, when dealing with biomedical signal processing problems, interpretability of the final outcome is paramount, especially when the results need to be communicated with clinicians and patients. Nowadays, deep learning methods such as artificial neural networks are rapidly gaining popularity. Although these models have been shown to give accurate results, they are black box models, meaning it is not straightforward to interpret how the outcome is calculated from the input. In contrast to artificial neural networks, tensor methods can lead to physiologically interpretable results. Tensor methods on the other hand can lead to interpretable components which can be physiologically interpreted. 


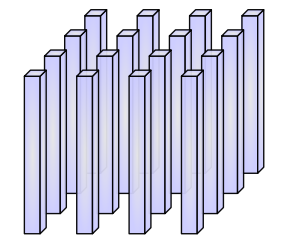

(a) Mode- 1 vectors or fibers.

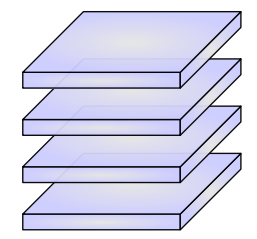

(b) Mode- $(2,3)$ slices.

Fig. 1: The mode-1 or column vectors and mode- $(2,3)$ or horizontal slices of a $4 \times 4 \times 4$-tensor. The other mode- $n$ vectors and mode- $(m, n)$ slices can be constructed in a similar way.

\section{Tensors and tensor tools}

\subsection{Basic concepts and notations}

Appropriate data representation is an important step that helps gain insight and process the data in an effective way. Generally speaking, data with $N$ modes are represented by a $N$ th-order tensor $\mathcal{X} \in \mathbb{R}^{I_{1} \times I_{2} \times \ldots \times I_{N}}$. The number of modes of a tensor is equal to the order, e.g. a tensor with three modes is a third-order tensor. Within this framework, it is also possible to define scalars $(N=0)$, vectors $(N=1)$, and matrices $(N=2)$.

Scalars, vectors and matrices are all commonly encountered in biomedical signal processing. A single observation or sample is represented by a scalar. Any signal recorded from a single source over a period of time can be described by a vector. The observations from acquisition with multiple sensors or electrodes are expressed in matrix-format. Data obtained from multiple subjects or due to repeated measurements can provide much more information, and these types of data can effectively be represented by a higher-order array or tensor.

From hereon, we refer to scalars with italic lower-case letters $x$, vectors with bold lower-case letters $\mathbf{x} \in \mathbb{R}^{I_{1}}$, and matrices with bold upper-case letters $\mathbf{X} \in \mathbb{R}^{I_{1} \times I_{2}}$. Finally, tensors are written as calligraphic letters $\mathcal{X}$. For example, a third-order tensor is denoted by $\mathcal{X} \in \mathbb{R}^{I \times J \times K}$ and its elements as $x_{i j k}$ with $i=1, \ldots, I, j=1, \ldots, J$ and $k=1, \ldots, K$.

A matrix $\mathbf{X}$ consists of row- and column vectors. In a similar way, mode-n vectors or fibers are defined for tensors. A mode- $n$ tensor fiber is the vector that is the result of fixing all indices except the $n$th index. For example, the mode- 1 fiber of a third-order tensor is analogue to a column vector and is denoted by $\mathbf{x}_{: j k}$. The mode- 2 and the mode- 3 fibers are the row and the tube vectors and are denoted by $\mathbf{x}_{i: k}$ and $\mathbf{x}_{i j}$ :, respectively. Figure 1a shows an example of the mode- 1 or column vectors of a third-order tensor. The mode- 2 and mode- 3 vectors can be visualized similarly. 


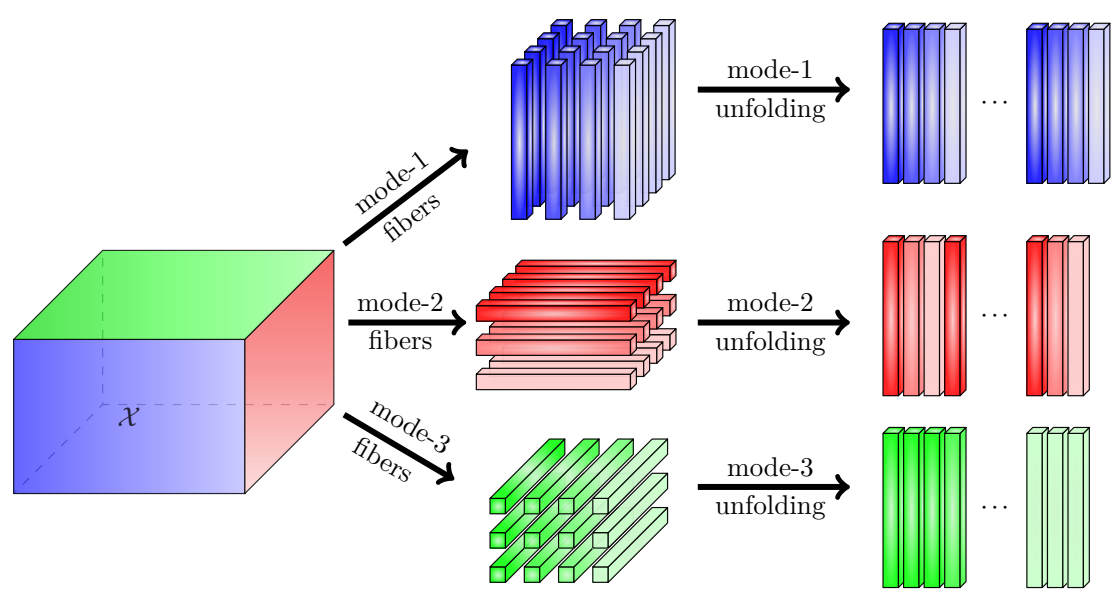

Fig. 2: Mode-1, mode-2, and mode-3 matricizations or flattenings or unfoldings of a 3 rd-order $(3 \times 4 \times 4)$ tensor.

Slices are defined in the same way. A mode- $(m, n)$ slice of a tensor is the matrix that is the result of fixing all indices except the $m$ th and $n$th index. In a third-order tensor, the different types of slices are typically named after their corresponding directions: this way horizontal (mode-(2,3)), vertical (mode- $(1,3)$ ) and frontal (mode- $(1,2)$ ) slices are defined. Figure $1 \mathrm{~b}$ shows the horizontal slices of a third-order tensor. The other slices can be visualized in a similar way.

The rank of a tensor is defined as the minimal number of rank- 1 tensors that generate the tensor as their sum. A rank-1 tensor is defined as the outer product of nonzero vectors.

Finally, the Frobenius-norm of a tensor $\mathcal{X} \in \mathbb{R}^{I_{1} \times I_{2} \times \cdots \times I_{N}}$ is given by

$$
\|\mathcal{X}\|_{F}=\sqrt{\langle\mathcal{X}, \mathcal{X}\rangle}=\sqrt{\sum_{i_{1}=1}^{I_{1}} \sum_{i_{2}=1}^{I_{2}} \cdots \sum_{i_{N}=1}^{I_{N}} x_{i_{1} i_{2} \cdots i_{N}}^{2}} .
$$

with $\langle\mathcal{A}, \mathcal{B}\rangle$ the representation of the inner product between two tensors. The Frobenius norm is analogous to the matrix Frobenius-norm and is essentially the multilinear generalization of the $L_{2}$-norm commonly used in vectors.

\subsection{Tensor operations}

In many applications, tensors are converted to matrices and vice versa. The transformation from a tensor to a matrix is referred to as tensor unfolding or flattening, a process where the elements of the tensor are reformatted in a 
lower-order structure [45]. One way of transforming tensors to matrices is the case of mode- $n$ unfolding or matricization, which places the mode- $n$ fibers of a tensor $\mathcal{X}$ as column-vectors in the matrix $\mathbf{X}^{(n)}$. The different unfoldings of a third-order tensor are presented in Figure 2.

When data or signals are naturally collected in matrix-format, they have to be transformed into tensors in order to be able to apply tensor methods. This is done through tensorization, where a vector or a matrix is mapped onto a tensor by creating additional modes. Many different tensorization methods exist, and the choice of method is dependent on both the data and the application. An overview of the most well-known deterministic and statistical techniques can be found in [21].

In the applications discussed in the remainder of the chapter, tensorization will be mainly done through segmentation, e.g. by dividing the signals in equal-length segments and stacking these in the frontal slices of a third-order tensor. The main advantage of ECG signals is that they contain 'natural' segments in the form of heartbeats or individual ECG waves, where the temporal profile of a beat is similar across all channels of the signal. Tensorizing them in such manner transforms ECG signals with modes time $\times$ channels to third-order tensors with modes time $\times$ channels $\times$ heartbeats, where each mode- 2 vector contains the temporal profile of one heartbeat in one channel. The resulting tensor allows to study the variations in ECG waveforms of consecutive heartbeats in all channels simultaneously, which is the goal in many ECG applications. Figure 3 illustrates the tensorization process: the two-dimensional multilead ECG signal that contains $J$ beats is transformed into a higher-order tensor.

An important tensor-matrix multiplication is the mode- $n$ product between a tensor $\mathcal{X} \in \mathbb{R}^{I_{1} \times I_{2} \times \ldots \times I_{N}}$ and a matrix $\mathbf{A} \in \mathbb{R}^{J \times I_{n}}$. It is represented as:

$$
\left(\mathcal{X} \cdot{ }_{n} \mathbf{A}\right)_{i_{1} i_{2} \cdots j \cdots i_{N}}=\sum_{i_{n}=1}^{I_{n}} x_{i_{1} i_{2} \cdots i_{n} \cdots i_{N}} a_{j i_{n}} .
$$

In practice, the mode- $n$ product multiplies each mode- $n$ vector of $\mathcal{X}$ with $\mathbf{A}$.

\subsection{Tensor decompositions}

Tensor decompositions are powerful tools that have been used successfully in various applications within signal processing and machine learning [75]. In this chapter, we will focus on the canonical polyadic decomposition (CPD)

and multilinear singular value decomposition (MLSVD) which have become popular tools in biomedical signal processing in recent years. 


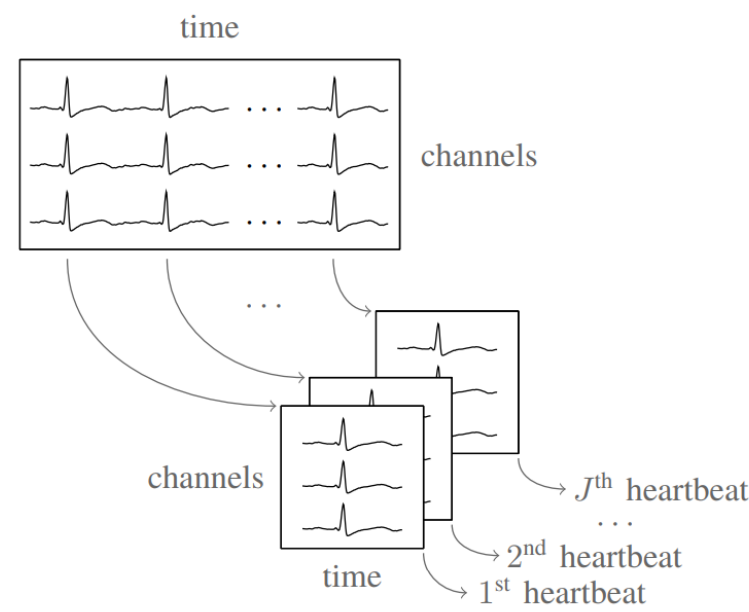

Fig. 3: Tensorization techniques create a tensor from a data matrix in a meaningful way, enabling tensor tools that allow one to extract more information [22]. Here, we apply a technique called segmentation to a multilead ECG data matrix, obtaining a third-order tensor with modes channels $\times$ time $\times$ beats. (Figure reproduced from [10].)

\subsubsection{Multilinear Singular Value Decomposition (MLSVD)}

The MLSVD is a powerful tensor tool in applications such as compression and dimensionality reduction in different fields; see [24, 45]. Recently, it has been applied for different ECG applications such as data compression and feature extraction for myocardial infarction classification, and irregular heartbeat classification $[63,65,10]$, which will be discussed in Section 4.

The key idea behind the MLSVD is to find the components that best capture the variations in each mode individually, while not considering the other modes at this point in time [45]. The MLSVD is the multilinear generalization of the singular value decomposition (SVD) for matrices [23].

As a recap, the SVD of a matrix $\mathbf{X} \in \mathbb{R}^{I \times J}$ can be written as:

$$
\mathbf{X}=\mathbf{U} \boldsymbol{\Sigma} \mathbf{V}^{T}=\sum_{i=1}^{R} \mathbf{u}_{i} \sigma_{i} \mathbf{v}_{i}^{T}
$$

where $\mathbf{U} \in \mathbb{R}^{I \times I}$ and $\mathbf{V} \in \mathbb{R}^{J \times J}$ are orthogonal matrices whose columns are respectively called the left and the right singular vectors of $\mathbf{X} . \boldsymbol{\Sigma} \in \mathbb{R}^{I \times I J}$ is a non-negative diagonal matrix that contains the ordered singular values $\sigma_{i}$ on the diagonal. The rank of the matrix $R$ is defined as the number of non-zero singular values. The SVD of a matrix $\mathbf{X}$ is often used when one 


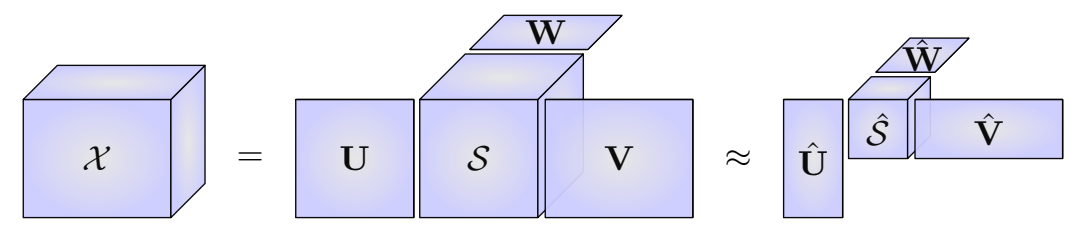

Fig. 4: The MLSVD and LMLRA of a third-order tensor $\mathcal{X}$. The column spaces of $\mathbf{U}, \mathbf{V}$, and $\mathbf{W}$ represent the signal subspaces along the three modes. The core tensor is non-diagonal, governing the interaction between the different modes.

wants to compute a low-rank approximation of $\mathbf{X}$, since it can be proven (in the Eckhart-Young theorem) that the optimal rank-r approximation of $\mathbf{X}$ is calculated by taking the singular vectors corresponding to the $r$ largest singular values of $\mathbf{X}$.

The MLSVD of a third-order tensor $\mathcal{X} \in \mathbb{R}^{I \times J \times K}$ is defined as follows [23]:

$$
\mathcal{X}=\mathcal{S} \cdot{ }_{1} \mathbf{U} \cdot \cdot_{2} \mathbf{V} \cdot 3 \cdot \mathbf{W}
$$

with $\mathbf{U} \in R^{I \times I}, \mathbf{V} \in R^{J \times J}$ and $\mathbf{W} \in R^{K \times K}$ orthogonal factor matrices that contain the mode- $n$ left singular vectors. The core tensor $\mathcal{S} \in \mathbb{R}^{I \times J \times K}$ governs the interaction between the different modes. It has the following properties:

- All-orthogonality: any two slices in a fixed mode are orthogonal.

- Ordening: the norms of the slices along any mode are ordered in decreasing manner

The Frobenius norms of the core tensor are called the multilinear or mode$n$ singular values (MSVs) of $\mathcal{X}$ and are denoted as $\sigma_{i}^{(n)}$. The values $I, J$, and $K$ correspond to the ranks of the different matrix unfoldings of $\mathcal{X}$ along the different modes.

The MLSVD can also be used to calculate a low multilinear rank approximation (LMLRA) of a tensor $\mathcal{X}$, but contrary to the matrix case it is not necessarily the optimal approximation $[23,17,75]$. Truncation can be done in a similar way as the matrix case, e.g. by only keeping the mode- $n$ singular vectors corresponding to the highest mode- $n$ singular values. While the LMLRA calculated in this way is not perfect, it is usually a good approximation since the multilinear singular values are ordered, meaning that most 'energy' is concentrated in the vectors corresponding to the first singular values (the part that is kept in the LMLRA). It is therefore considered a suitable solution for most applications, which can be refined by iterative algorithms if necessary [42].

To have the core tensor $\mathcal{S}$ as a compressed form of $\mathcal{X}$, reduced dimensions of different modes can be chosen as $R_{1} \ll I, R_{2} \ll J$ and $R_{3} \ll K$. Hence, $\mathcal{X}$ can be approximated as 


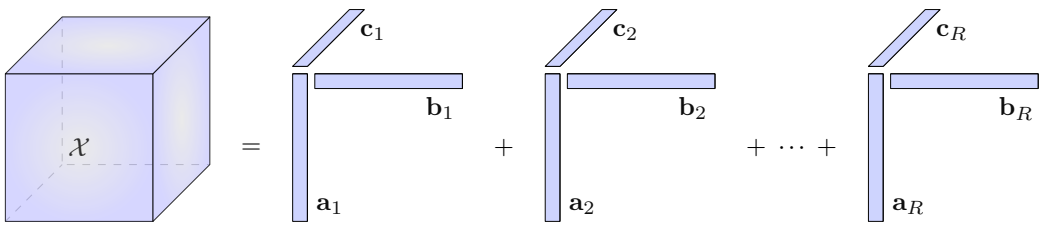

Fig. 5: Schematic representation of the Canonical Polyadic Decomposition of a third-order tensor in the individual factor vectors $\mathbf{a}_{r}, \mathbf{b}_{r}$ and $\mathbf{c}_{r}$ for $r=1, \ldots, R$. The different factor vectors can be used in various ways depending on the application.

$$
\mathcal{X} \approx \hat{\mathcal{S}} \cdot{ }_{1} \hat{\mathbf{U}} \cdot 2 \hat{\mathbf{V}} \cdot 3 \cdot \hat{\mathbf{W}}
$$

where $\hat{\mathcal{S}} \in \mathbb{R}^{R_{1} \times R_{2} \times R_{3}}, \hat{\mathbf{U}} \in \mathbb{R}^{I \times R_{1}}, \hat{\mathbf{V}} \in \mathbb{R}^{J \times R_{2}}$ and $\hat{\mathbf{W}} \in \mathbb{R}^{K \times R_{3}}$, respectively.

\subsubsection{Canonical Polyadic Decomposition (CPD)}

A polyadic decomposition ( $\mathrm{PD}$ ) expresses a tensor as a sum of rank-one components. If the number of components is the minimal number of components necessary to exactly decompose the tensor, then the PD is called the canonical polyadic decomposition (CPD). For a third-order tensor $\mathcal{X} \in \mathbb{R}^{I \times J \times K}$, it is expressed as:

$$
\mathcal{X}=\sum_{r=1}^{R} \mathbf{a}_{r} \otimes \mathbf{b}_{r} \otimes \mathbf{c}_{r}
$$

with $R$ the total number of components or rank of the decomposition and $\mathbf{a}_{r} \in \mathbb{R}^{I}, \mathbf{b}_{r} \in \mathbb{R}^{J}$ and $\mathbf{c}_{r} \in \mathbb{R}^{K}$ for $r=1, \ldots, R$ the factor vectors. CPD is also known as PARAFAC (Parallel Factor Analysis) or CANDECOMP (Canonical Decomposition) [45]. A schematic presentation of CPD for a third-order tensor is shown in Figure 5.

While the definition of tensor rank is straightforward, its determination is a NP-hard [36, 39]. Different approaches for automatic rank estimation exist such as the core consistency diagnostic [14] or Rankest available in Tensorlab [81]. Both approaches gradually increase the tensor rank and compare the results of the decomposition with the original tensor. They however lead to an overestimation of the tensor rank for noisy signals [18], and are therefore less suitable for biomedical signals which inherently contain noise. In practice, visual inspection of the obtained components for different ranks is very informative for evaluating the results and determining suitable rank values. Another starting point are the multilinear singular values, which can provide a lower-bound for determining the rank [17]. 
One of the main advantages of tensor decompositions compared to matrixbased methods is that the resulting components are unique under mild conditions (up to trivial permutation and scaling indeterminacies). A general framework for uniqueness properties of third-order tensors is presented in $[25,26,27]$. This means that no additional constraints have to be imposed on the data or factor matrices which is required for matrix decompositions such as orthogonality in case of PCA or independence in ICA.

While many other tensor decomposition methods exist, CPD can be considered one of the most accessible approaches. Generally, it generates components which can be easily visualized and interpreted. Furthermore, different computation methods have been developed that deal with practical issues such as missing data [86] or noisy signals [11]. In the next Section, these methods will be applied to identify different ECG features, highlighting the power of these tensor decompositions in cardiac applications.

\section{Applications of Tensors in ECG Processing}

Multilead ECG signals are described by two parameters: time and space. In contrast to other biomedical signals, such as EEG, ECG is relatively noise-free and exhibit intrinsic structure, making this type of data a prime candidate for tensor-based analysis. In the last few years, tensor methods have been used in a limited number of ECG applications.

This section gives an overview of five typical problems in ECG processing where tensors have been used successfully to obtain relevant results:

1. ECG data compression [63],

2. Detection and localization of myocardial infarction [65],

3. Irregular heartbeat classification [30, 10],

4. Detection and quantification of T-wave alternans [29, 31, 32, 67],

5. Analysis of changes in heartbeat morphology [33].

The goal of this section is to highlight the flexible nature in which tensor tools can be used in various important use-cases. We also show how the tensor decompositions can be adapted to different typical characteristics of ECG signals in order to deal with real-life problems such as noisy signals or changes in heart rate. For a more comprehensive explanation of the methods, we refer to the dedicated papers of each method.

Many different tensor decompositions exist, each with specific advantages and disadvantages. The applications discussed here all make use of either the MLSVD or CPD to decompose the ECG signal. The first two applications combine the MLSVD with the discrete wavelet transform to exploit different types of correlations in the ECG signal. For the next two applications, e.g. irregular heartbeat classification and detection of $\mathrm{T}$ wave alternans, we present and compare a CPD- and MLSVD-approach. Both approaches have their own 
strengths and are preferred in different cases. Finally, the last application is tackled using another variant of the CPD.

\subsection{ECG data compression}

Diagnosis of heart diseases often requires long-term multilead ECG recordings in order to accurately determine the extent of cardiac abnormalities. When dealing with many patients in a hospital setting, this quickly leads to large-scale datasets: a 24-hour ECG signal measured with 12 leads with a sampling frequency of $250 \mathrm{~Hz}$, digitized at 11 bits/sample for example requires a storage capacity of $2.88 \mathrm{~Gb}$. There is a growing need to store these datasets as compactly as possible without distorting the ECG signals, since this might lead to the loss of important clinical information.

For this purpose, many ECG compression methods have been developed that reduce the number of samples required to store the signal. They mostly start from the idea that there are different types of correlations present in ECG signals [43]:

1. intra-beat: correlations between the different samples in a heartbeat,

2. inter-beat: correlations among different heartbeats in a long-term signal,

3. inter-lead: correlations between different leads in a multilead signal.

Different methods exploit one or more type(s) of correlations in order to efficiently reduce the required storage capacity. Vector-based methods utilize intra-beat correlations by means of sampling or interpolation $[1,19,43,59]$. Matrix-based methods can handle up to two types of correlations simultaneously. Different matrix decompositions, such as the Karhunen-Loève transform (KLT) or PCA [73] and SVD have been used for ECG compression[88, 64]. The use of tensor methods allows analysis of all three correlations types at the same time, making optimal use of all information present in the signal.

In the remainder of this section, we will describe a multiscale tensor-based ECG compression method that uses the discrete wavelet transform (DWT) to represent the signal. It starts from an ECG signal that has been tensorized by segmenting the signal in different heartbeats, as described in Section 3 and shown in Figure 3. A full description of the method can be found in [65].

The ECG signal is a low-frequency signal where most of the physiological activity is contained in the frequency band between 1 and $40 \mathrm{~Hz}$. It furthermore has different morphological components (in the form of the different ECG waves) that vary both in duration and frequency. The multilevel decomposition using DWT is able to grossly segment the different components into different subbands while preserving the information in an effective way [55]. An $L$-level wavelet decomposition splits up the ECG signal in $L+1$ subbands of different dimensions. Here, a decomposition of $L=7$ levels is applied on the mode-2 fibers $\mathbf{x}_{i: k}$ of the original tensor $\left(\mathcal{X} \in \mathbb{R}^{I \times J \times K}\right)$ with 
modes channels $\times$ time $\times$ beats. Each mode- 2 fiber represents one heartbeat in one channel. The result is eight subband tensors: one approximation subband tensor $\mathcal{A}_{L}$ containing the approximation coefficients in mode-2, and $L$ detail subbands tensors $\mathcal{D}_{l}, l=L, \cdots, 1$ that contain the detail coefficients in mode- 2 of respective levels. The dimensions of these tensors, $\mathcal{A}_{L}$ and $\mathcal{D}_{l}, l=L, \cdots 1$, are given as $I \times J / 2^{L} \times K$ and $I \times J / 2^{l} \times K$, respectively, and we say now each subband tensor has modes channels $\times$ wavelet coefficients $\times$ beats. Because the different heartbeats in the ECG signal are related in time and in space (due to inter-beat and inter-lead) correlations, the wavelet coefficients show a high correlation in the different subbands [66]. Therefore they can be represented in a more efficient way with the truncated MLSVD.

Following (4), the MLSVD of each subband tensor can be expressed as

$$
\begin{aligned}
\mathcal{A}_{L} & =\mathcal{S}_{A_{L} \cdot{ }_{1}} \mathbf{U}_{A_{L} \cdot{ }_{2}} \mathbf{V}_{A_{L} \cdot{ }_{3}} \mathbf{W}_{A_{L}} \\
\mathcal{D}_{l} & =\mathcal{S}_{D_{l} \cdot{ }_{1}} \mathbf{U}_{D_{l} \cdot 2} \mathbf{V}_{D_{l} \cdot 3} \mathbf{W}_{D_{l}}
\end{aligned}
$$

where $\mathbf{U}_{A_{L}}, \mathbf{V}_{A_{L}}$, and $\mathbf{W}_{A_{L}}$ are orthonormal matrices of approximation subband tensors, and $\mathbf{U}_{D_{l}}, \mathbf{V}_{D_{l}}$, and $\mathbf{W}_{D_{l}}$ are that of each details subband tensor, along different modes. The orthonormal column vectors of $\mathbf{U}_{A_{L}}$ and $\mathbf{U}_{D_{l}}$ span the channel space of each subband. Similarly, the column vectors of $\mathbf{V}_{A_{L}}, \mathbf{V}_{D_{l}}$ and $\mathbf{W}_{A_{L}}, \mathbf{W}_{D_{l}}$ span the coefficient space and heartbeat space.

The ECG signal is then compressed by truncating the MLSVDs of the subband tensors that contain the detail coefficients. The truncation is done such that $95 \%$ of the energy contained in the multilinear singular values in each mode is kept. This means that for each subband tensor $\mathcal{D}_{i}$ we will define a multilinear rank $\left(R_{1}, R_{2}, R_{3}\right)$ such that:

$$
\frac{\sum_{i=1}^{R_{n}}\left(\sigma_{i}^{(n)}\right)^{2}}{\left\|\mathcal{S}_{D_{l}}\right\|_{F}^{2}} \geq 0.95, \quad n=1,2, \text { and } 3 .
$$

where $\left\|\mathcal{S}_{D_{l}}\right\|_{F}$ is the Frobenius norm of each detail subband tensor. Note that the multilinear rank of the truncated MLSVD $\left(R_{1}, R_{2}, R_{3}\right)$ is defined separately for each subband tensor $\mathcal{D}_{l}$. Since the multilinear singular values typically decrease rapidly for most subbands, values for $R_{n}$ can be kept relatively low resulting in an efficient compression. While it is known that the truncated MLSVD in general does not result in the optimal low-rank approximation of the original tensor, the results are however considered good enough for most applications.

In order to compare different vector-, matrix- and tensor-based approaches we use standard measures such as the compression ratio $(\mathrm{CR})$, which is defined as

$$
C R=\frac{\text { bits in the input signal }}{\text { bits in the compressed signal }} .
$$

The higher the CR, the better the compression performance. However, as mentioned before, this should not come at the cost of reduced signal accuracy. 
Table 1: Comparison of tensor-based ECG compression method with vectorand matrix-based methods using the PTB database [69]. The tensor-based method using multilevel DWT and MLSVD in [65] exploits all three types of correlations and leads to the best results. Adapted from [63]

\begin{tabular}{llcc}
\hline Decomposition & Correlation type & CR & PRD (\%) \\
\hline KLT [16] & intra-beat & 7.25 & 3.18 \\
DWT + PCA [73] & inter-lead & 12.61 & 2.66 \\
SVD [88] & intra- and inter-beat & 15.70 & 2.83 \\
DWT + SVD [63] & intra-beat, inter-lead & 19.34 & 3.05 \\
DWT + MLSVD [65] intra- and inter-beat, inter-lead 45.00 & 2.71 \\
\hline
\end{tabular}

To control this trade-off, we therefore use additional quality measures such as the percentage of root-mean-square difference (PRD). The PRD is defined as

$$
P R D=\sqrt{\frac{\sum_{n=1}^{N}(x(n)-\tilde{x}(n))^{2}}{\sum_{n=1}^{N} x(n)^{2}}} \times 100
$$

where $N$ is the total number of samples and $x(n)$ and $\tilde{x}(n)$ are, respectively, the original and the reconstructed signals. The quality of the reconstructed signal is 'very good' and 'good' if the PRD (\%) value is in between 0 and 9. Analysis on different compression algorithms however suggests to keep the PRD value as small as possible (under 3\%) [56].

Table 1 shows CR and PRD values for several of the methods mentioned in the first part of this section together with the results for the proposed tensorbased method. In order to make a fair comparison possible, all methods were performed in the same environment and on the same database, the PTB database available on Physionet [69]. From Table 1 it is clear that increasing the number of correlation types that are exploited by the decomposition results in a better compression ratio: the lowest $\mathrm{CR}$ values are obtained by methods using only one type of correlation. Adding an additional correlation type immediately improves the compression ratio with minimal impact on the PRD. The proposed tensor-based method that combines DWT and MLSVD results in the highest compression ratio with $\mathrm{PRD}<3$.

Here, the combination of multiscale DWT and truncated MLSVD was used solely for data compression. The technique could however also be used for feature extraction, since the singular values and singular vectors inherently contain valuable information about the underlying ECG signal. 


\subsection{Myocardial infarction classification}

Myocardial infarction (MI) occurs when the flow of oxygen-rich blood to the heart muscle is halted, which causes cell death and tissue damage. It can be diagnosed through blood tests or imaging techniques [82]. However, since $\mathrm{MI}$ is also known to cause alterations in the electrical conduction pattern of the heart, efficient detection can also be done by examining changes in the ECG signal (such as ST-segment deviations or T wave inversions). MI detection should be done as early as possible, so accurate treatment can start and damage to the heart muscle can be kept minimal. Therefore there is a clear advantage over using ECG analysis over other techniques, since results can be processed and analyzed in real-time.

A second objective is often MI localization, where the goal is to indicate the location on the heart wall that is affected by the infarction. This can be done by making use of the fact that each ECG lead represents cardiac activity from a different spatial angle, as discussed earlier. Clinically, we make the distinction between lateral leads (I, aVL, V5 and V6), anterior leads (V3V4), septal leads (V1-V2) and inferior leads (II, III, aVF). When a patient experiences an anterior MI for example, it will mainly be characterized by changes in the anterior leads. This way, we can define four major MI types: anterior MI (AMI), inferior MI (IMI), posterior MI (PMI) and lateral MI (LMI). Additional subtypes are possible when the MI can be detected in more than one region, such as for example antero-lateral MI (ALMI), anteroseptal MI (ASMI) or infero-lateral MI (ILMI).

Since MI detection and localization is an important clinical task, a large number of automated methods have been developed for this purpose. Their goal is to identify discriminative features that can characterize the different MI subtypes compared to ECG signals of healthy subjects. Many methods aim to detect and quantify specific ECG changes such as ST-segment deviations $[53,5,80]$ or alterations in the QRS complex [71]. While these features are known to change after MI, they are difficult to accurately measure in the presence of noise, which may affect the final performance. A second class of methods therefore transforms the ECG signal to the frequency- or wavelet domain before feature extraction. Examples can be found in $[44,74]$, where the DWT is used to represent the signal and extract more robust features. This way detection and localization accuracies of $>95 \%$ can be achieved. A comprehensive view of recent advances in the field can be found in [2].

Similar to ECG compression, tensor decompositions can exploit spatial and temporal correlations in the signal simultaneously to detect and localize the MI in an even more efficient way. The method described here is a multiscale approach that starts from an ECG signal that has been converted to the multiscale domain and compressed with the method described earlier. Here however, features are extracted from the MLSVD to detect and localize MI.

MI induces morphological changes in the ECG signal which are captured by the multilinear singular values (MSVs) of the subtensors corresponding to 

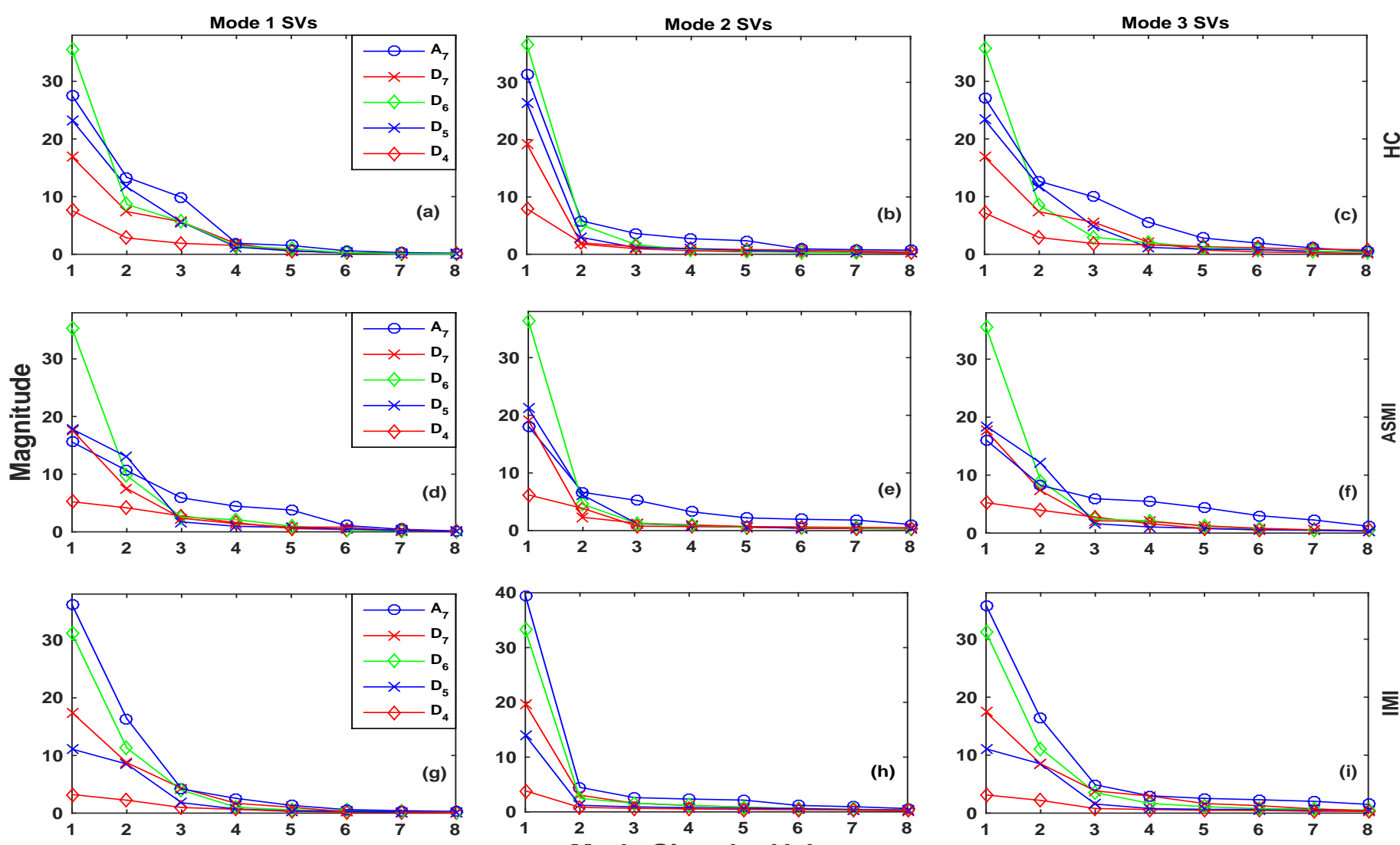

Fig. 6: The multilinear singular values for a healthy subject (top row), a subject with an ASMI (middle row) and an IMI (bottom row). The myocardial infarction causes changes in the frequency content of the ECG signal and induces changes in the MSVs of the different subband tensors. Reproduced from [65]. 
Table 2: Comparison of MI detection and localization performance with existing methods. Adapted from [65]

\begin{tabular}{|c|c|c|c|c|c|}
\hline \multirow[b]{2}{*}{ Method } & \multirow[b]{2}{*}{ Decomposition } & \multicolumn{3}{|c|}{ Detection } & \multirow{2}{*}{$\frac{\text { Localization }}{\operatorname{Acc}(\%)}$} \\
\hline & & $\operatorname{Sen}(\%)$ & Spe (\%) & $\operatorname{Acc}(\%)$ & \\
\hline Arif [5] & n.a. & 97.2 & 99.6 & $\mathrm{NA}$ & 98.8 \\
\hline Sharma [74] & $\mathrm{DWT}+\mathrm{PCA}$ & 93.0 & 99.0 & 96.0 & 99.6 \\
\hline Proposed & $\mathrm{DWT}+\mathrm{MLSVD}$ & 94.6 & 96.0 & 95.3 & 98.1 \\
\hline
\end{tabular}

the different wavelet bands. An illustration is shown in Figure 6, which shows the MSVs for a healthy subject (top row), a patient with an ASMI (middle row) and a patient with an IMI (bottom row) respectively. In a healthy subject, most of the energy is contained in the $\mathcal{D}_{6}$ subband tensor, followed by $\mathcal{A}_{7}, \mathcal{D}_{5}$ and $\mathcal{D}_{7}$. This pattern is visible in all three modes. The explanation can be found in the QRS complex, which is the most dominant wave in healthy ECG signals and which is mainly segmented in $\mathcal{D}_{6}$. ASMI introduces pathological Q-waves in the ECG signal, which changes the frequency content in different wavelet bands. In the MSVs, this corresponds to a large drop of the values for $\mathcal{D}_{5}$ and $\mathcal{A}_{7}$. Similarly, an ISMI causes ST-elevation which increases the MSVs for the $\mathcal{A}_{7}$ subband. The significant MSVs of modes 1 and 2 were therefore used as features for MI detection, whereas the mode $3 \mathrm{MSVs}$ are used to localize the MI. Additionally, normalized multiscale wavelet energy features were also extracted to make MI localization more accurate. A more comprehensive explanation of the different features can be found in [65].

The features are then used as input to a support vector machine classifier with $\chi^{2}$-kernel, to do the classification in a supervised way. The SVM parameters (both the regularization parameters and kernel parameters) are optimized using a grid-search algorithm and 5-fold cross-validation on the training set. The final performance is calculated on an independent test set. The performance of the proposed method is evaluated on a subset of the PTB database [69]. The full dataset considered here consists of 390 records: 78 healthy controls and 312 records of subjects with different types of MI. 89 records were used as training set, the remainder as test set.

The results of the methods mentioned in Table 2 are rather similar for all three methods. The proposed tensor-based approach however has several advantages. First of all, the results on the first two methods were not obtained with an independent test set (e.g. which does not contain signals of patients in the training set). There is therefore a significant risk of over-fitting, meaning that the methods are not capable of generalizing and will lead to inferior results on different datasets. Second, the matrix-based features are calculated for each lead individually, which is computationally not very efficient, while the proposed tensor-based approach processes all leads simultaneously. The tensor-based method, on an average, takes $0.23 \mathrm{~s}$ and $0.021 \mathrm{~s}$ for detection 
and localization, respectively, during testing compared to $0.44 \mathrm{~s}$ and $0.025 \mathrm{~s}$ of [74].

\subsection{Heartbeat classification}

Cardiac arrhythmias affect millions of people and are the main cause of many cases of sudden cardiac death. A large number of people could benefit from a better and more reliable detection of cardiac dysfunction. An arrhythmia is defined as any disturbance of the normal sinus rhythm. This can be a perturbation or abnormality in the rate, the regularity, the site of origin or the conduction of the electrical impulses of the heart. In general, they can be divided in two main groups: the first group, morphological arrhythmia, contains arrhythmia that consist of only one heartbeat; the second group consists of arrhythmia formed by a group of heartbeats and is called rhythmic arrhythmia [52]. The focus of this section lies in automatic detection of the first class, e.g. morphological arrhythmia. Various types of morphological arrhythmia exist, each differing in the origin and/or electrical pathway of the abnormal beat. In general, we make the distinction between three types of abnormal beats: Normal beats, atrial or supraventricular beats and ventricular beats. Normal beats refer to beats that originate during normal sinus rhythm. Atrial and ventricular beats respectively arise in the atria or ventricles.

Classification of heartbeats in different classes is important for two main reasons. Firstly, heart rate variability studies, which are useful in many clinical and non-clinical scenarios require series of normal RR-intervals. The presence of ectopic beats or other abnormalities introduces abrupt changes in the RR time series which can seriously disturb the values of HRV indices. Secondly, most ventricular arrhythmia, which are potentially lethal, are initiated by premature ventricular beats. Their frequency and complexity have been shown to be predictive for predicting SCD in certain patient groups and have been used as stratification tools in large clinical trials.

Here we present two different tensor-based approaches to irregular heartbeat detection. The first method detects irregular heartbeats in an unsupervised manner using the CPD. A full explanation can be found in Goovaerts et al. [30]. The second method uses the MLSVD to perform a supervised classification and is described in Boussé et al. [10]. For both methods, the multilead ECG signal is first preprocessed to remove major noise sources such as baseline wander and high-frequency noise. The signal is then transformed into a third-order tensor in the same way as shown in Figure 5. First, we segment the signals in individual heartbeats of equal lengths. Next, all heartbeats are stacked in the third mode, resulting in a third-order tensor $\mathcal{T}$ with dimensions channel $\times$ time $\times$ heartbeat. Tensorizing the signals in this way allows us to study the differences between the heartbeats in a signal in a straightforward way by studying the variation in the third mode. 

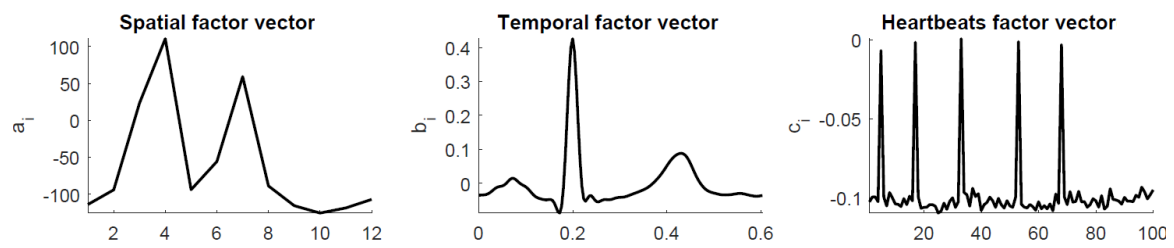

Fig. 7: The factor vectors for the different modes obtained by CPD. The irregular heartbeats are clearly distinguishable in the third factor vector and can be detected by thresholding or clustering. Reproduced from [34].

\subsubsection{CPD}

We explained in Section 3 that the CPD can be used to decompose a tensor in a sum of rank- 1 components. In this case, $R$, the rank of the decomposition, is fixed to one since we are interested in the main variation between different heartbeats. The result is 1 rank-one tensor consisting of three loading vectors $\mathbf{a}_{1}, \mathbf{b}_{1}$ and $\mathbf{c}_{1}$.

An example of the CPD result for a 12-lead ECG signal with five abnormal heartbeats is shown in Figure 7. The feature vectors have a straightforward physiological interpretation, especially for the temporal and heartbeat mode. The first loading vector, $\mathbf{a}_{1}$, corresponding to the channel mode, is associated with the differences in heartbeat morphologies over different channels. The second loading vector $\mathbf{b}_{1}$ (time) shows the temporal profile of the component and is a representation of the average heartbeat in the signal. It is easily recognizable as a normal (regular) heartbeat, which is expected since the majority of heartbeats are by definition normal. Furthermore the morphology of a normal heartbeat is similar over all channels, making it suitable to capture in a rank-one component. Finally, the factor vector of the heartbeat dimension is the most important for irregular heartbeat detection since it shows the changes in the ECG over different heartbeats. The values of this loading vector for normal heartbeats varies around -0.1 . The abnormal heartbeats are easily distinguishable by their higher values, and they can be detected by simple thresholding or clustering methods.

\subsubsection{MLSVD}

The second approach uses the MLSVD to detect heartbeats in a supervised way, e.g. by making use of a training set of heartbeats with known label. We first collect all labeled training beats in a tensor $\mathcal{T}$. The (truncated) MLSVD of this tensor is given by:

$$
\mathcal{T}=\mathcal{S} \cdot{ }_{1} \mathbf{U}_{c} \cdot{ }_{2} \mathbf{U}_{t \cdot 3} \mathbf{U}_{3}
$$


where $\mathbf{U}_{c}, \mathbf{U}_{t}$ and $\mathbf{U}_{h}$ form an orthonormal basis for the spatial, temporal and heartbeats components respectively. Every heartbeat in a particular channel $\mathbf{t}$ can be written as:

$$
\mathbf{t}^{\mathrm{T}}=\mathcal{S} \cdot{ }_{1} \mathbf{C}_{c}^{T} \cdot{ }_{2} \mathbf{U}_{t} \cdot{ }_{3} \mathbf{c}_{h}^{T}
$$

The mode- 2 unfolding of this Equation is a so-called Kronecker-product equation (KPE), which is a linear system with a solution that has a Kronecker product structure. It expresses each heartbeat in the column space $\mathbf{U}_{t}$ and an unfolded interaction tensor $\mathcal{S}$ :

$$
\mathbf{t}=\mathbf{U}_{t} \mathbf{S}_{(2)}\left(\mathbf{c}_{h} \otimes \mathbf{c}_{c}\right)
$$

We can solve this system in an efficient way to obtain coefficient vectors $\mathbf{c}_{h}$ and $\mathbf{c}_{c}$ using nonlinear least squares algorithms [11].

A new heartbeat without known label, that is thus not included in $\mathcal{T}$, can now be classified by solving (10) as such:

$$
\mathbf{U}_{t} \mathbf{S}_{(2)}\left(\mathbf{c}_{h}^{(\text {new })} \otimes \mathbf{c}_{c}^{(\text {new })}\right)=\mathbf{t}^{(\text {new })}
$$

to obtain estimates $\hat{\mathbf{c}}_{h}^{(\text {new) }}$ and $\hat{\mathbf{c}}_{c}^{(\text {new) }}$. The new heartbeat can then be classified by comparing $\hat{\mathbf{c}}_{h}^{(\text {new })}$ with the rows of $\mathbf{U}_{h}$, after normalizing all vectors to correct for scaling and sign differences. Since each row of $\mathbf{U}_{h}$ corresponds to a heartbeat from the training set with known label, we can assign the new heartbeat the label corresponding to the closest match.

Note that while the data of all channels are used to compute the MLSVD, classification is only done using the signal from a single channel. In practice, however, the MLSVD model holds only approximately and incorrect classification can possibly occur. It is therefore possible to make the classification more robust by using heartbeats from multiple channels which can be solved using a coupled KPE. More information can be found in [10].

To compare both approaches with each other and with alternative matrixbased approaches, they were both applied on the first ten signals of the INCART database, which is a publicly available multilead database that contains heartbeat annotations. The unsupervised CPD-based method obtains a mean $F_{1}$-score of $79 \%$. Further inspection of the results however revealed that the method distinguishes normal and ventricular beats almost perfectly, but lacks performance for atrial beat classification. The MLSVD-based method reaches a mean $F_{1}$-score of $94.2 \%$ which is better than the best performance (92\%) of traditional techniques [52]. The results could be improved further by using all channels to perform the classification. Furthermore, because of the generic nature of the method [11], the same concept can be applied for other classification problems with minimal alterations, which shows the flexibility of the method. Examples of recently developed applications in biomedical signal processing are detection of atrial fibrillation [28] and EEG classification $[84]$. 
The CPD-based approach is clearly not suitable as general irregular heartbeat detection method, but could be used to automatically detect ventricular beats. Since most lethal arrhythmia are initiated by premature ventricular contractions, a type of ventricular beats, this class is more important in a clinical context so this would be a practically useful result. It is furthermore an unsupervised detection method, meaning that no annotations are required to perform the analysis, which is an advantage in clinical practice. Similarly to the proposed MLSVD-based approach, the CPD-based method can be used to analyze other abnormal ECG patterns with minimal alterations. In the next Section for example, we present a similar algorithm to analyze $\mathrm{T}$ wave alternans.

\subsection{T-wave alternans detection and estimation}

T wave alternans (TWA) is an abnormal ECG pattern where the amplitude of the $\mathrm{T}$ wave shows a beat-to-beat change in a characteristic ABABABpattern [60]. It can be detected in healthy hearts at high heart rates, but if it also appears at lower heart rates $(<110$ beats per minute) it is a sign of electrically unstable tissue and associated with increased mortality [41]. When the variation between subsequent $T$ waves is large enough, TWA can be detected by visually inspecting the ECG signal. In cases where the difference is only a few microvolts or when dealing with long ECG signals this is however not feasible. Therefore (semi-)automatic methods are used that can reliably detect TWA in the ECG signal.

Martínez and Olmos [54] gave an overview of commonly used algorithms for (semi-)automatic T Wave Alternans detection. Most methods start with a preprocessing stage followed by the actual TWA detection. Examples of commonly used methods are the modified moving average method [61] or spectral method [78]. Most methods decompose the ECG signal to a beat-tobeat time series that contains the $\mathrm{T}$ wave characteristics. The actual detection of $\mathrm{T}$ wave alternans is then done on this decomposed signal. Most TWA detection and quantification methods have been designed for use with single lead ECG signals. They can be applied on multilead signals by processing each lead individually and combining the results of different leads in a second stage, however it has been shown that this might not be optimal [15]. Analyzing all leads simultaneously can be done in a straightforward way through the use of tensors, as we already showed in the previous examples. Here, we present two different tensor-based approaches for TWA detection and quantification. Similarly to the previous section, the first method starts from the CPD [29, $31,32]$ and the second uses the MLSVD as main building block [67]. 


\section{CPD and PARAFAC2}

In a similar way as before, the ECG signal was transformed into a tensor before applying tensor decompositions. Since the objective is TWA detection, the tensor should be constructed in such a way that the changes in $\mathrm{T}$ waves over different heartbeats are maximally emphasized. Therefore we constructed a $\mathrm{T}$ wave tensor $\mathcal{T}$ : a third-order tensor that consists of the $\mathrm{T}$ waves of all channels and heartbeats.

There is an important difference between the tensorization in the previous Section and here: before, the individual heartbeats were segmented by selecting a window around the $\mathrm{R}$ peaks. When these heartbeats are placed in the tensor, the different heartbeats are automatically synchronized at the $\mathrm{R}$ peak location (which is always at the same time location in the segmentation window). Here, segmentation is done by taking a fixed-size window after the $\mathrm{R}$ peak that contains the $\mathrm{T}$ wave. When for example the heart rate changes within a signal, it is possible that the timing of the $\mathrm{T}$ wave with respect to the $\mathrm{R}$ peak changes, and that the $\mathrm{T}$ waves of different beats are thus not strictly aligned in the third mode.

Similarly to the approach for irregular heartbeat detection, CPD was the first decomposition method used to break down the tensor in different factors. Since here the interest lies in the main variation between subsequent $\mathrm{T}$ waves, only one component was extracted and therefore $R$ was restricted to one. If the tensor is low-rank, the CPD model is accurate and the three factor vectors will capture the main variations present in the tensor. When the ECG signal is relatively noise-free and the $T$ waves are perfectly aligned in the third mode, this assumption is met and CPD will lead to correct results. When there is however a variation in for example the timing of subsequent $\mathrm{T}$ waves (as can be the case with a changing heart rate), the tensor cannot be decomposed in rank-1 components and CPD will lead to inaccurate results. In those cases it is better to use a more general tensor decomposition method such as PARAFAC2. PARAFAC2 is a tensor decomposition method that is a variation of the standard CPD [13]. The main difference is that PARAFAC2 is less restrictive than $\mathrm{CPD}$ in the sense that it allows variations in the factor vectors of one mode. This is especially useful when one of the factors contains a time shift. PARAFAC2 models an individual loading vector $\mathbf{b}_{1, m}$ for each frontal slice of the tensor, effectively making $\mathbf{B}_{1}$ a loading matrix that contains the collection of loading vectors for each heartbeat. Each row of this loading matrix corresponds to the temporal profile of a $\mathrm{T}$ wave in one heartbeat, taking into account the possible time shifts in $\mathrm{T}$ waves due to heart rate changes.

The result of the tensor decomposition is a collection of three factor vectors (or two factor vectors and one matrix in the case of PARAFAC2), which give information about the structure of the signal in the three tensor modes. All three components give valuable information about the $\mathrm{T}$ wave characteristics in time and space, but since the mode- 3 factor vector expresses the differences 


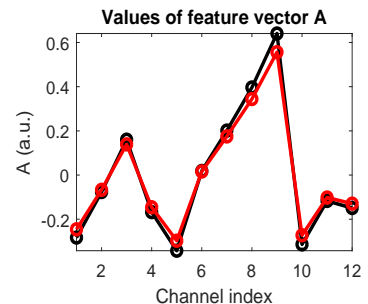

(a) Spatial vector (no TWA)

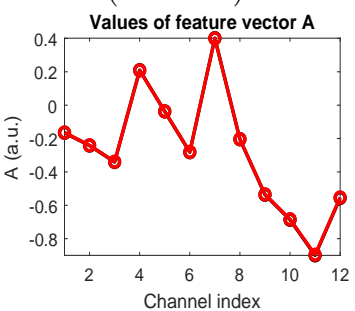

(d) Spatial vector (TWA)

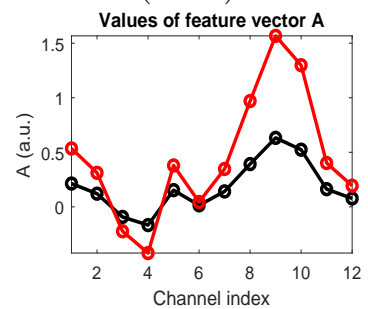

(g) Spatial vector (TWA + shift)

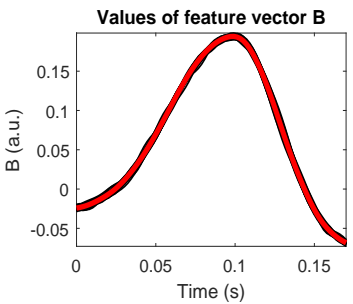

(b) Temporal vector (no TWA)

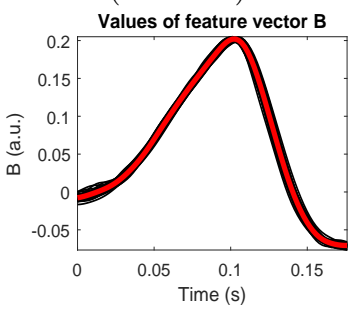

(e) Temporal vector (TWA)

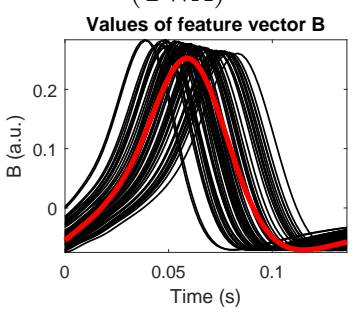

(h) Temporal vector (TWA + shift)

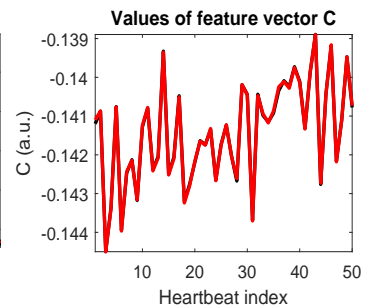

(c) Heartbeats vector (no TWA)

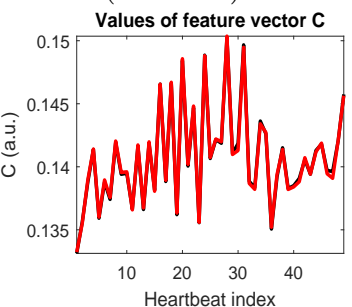

(f) Heartbeats vector (TWA)

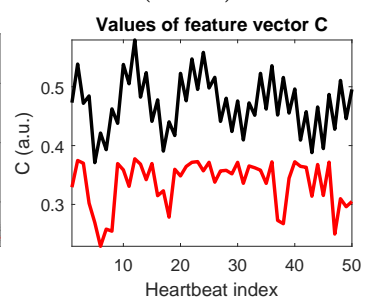

(i) Heartbeats vector (TWA + shift)

Fig. 8: Vectors $\mathbf{a}, \mathbf{b}$ and $\mathbf{c}$ for signal without TWA (top row), a signal with TWA (middle row) and a signal with TWA and shifted T waves (bottom row). The results for CPD are plotted in red, the results for PARAFAC2 in black. The three different columns show respectively the spatial, temporal and heartbeats feature vectors. PARAFAC2 is better able to capture dynamic changes in the ECG signal caused by changes in heart rate. When such differences are present, CPD fails to accurately extract TWA activity. Reproduced from [32] 
in the $\mathrm{T}$ wave in subsequent heartbeats, only this vector was used for the actual detection of $\mathrm{T}$ wave alternans. If a signal contains TWA, the typical ABABAB-pattern that is present in the $\mathrm{T}$ wave amplitude will be captured by the tensor decomposition as an alternating time series in the third dimension. To detect and quantify TWA the number of consecutive turning points of $\mathbf{c}_{\mathbf{r}}$, the loading vector of the heartbeat dimension, were first detected. The total TWA level in a signal is then defined as the mean amplitude difference between all consecutive turning points.

To illustrate the difference between the decomposition methods, Figure 8 shows the CPD and PARAFAC2 results for three signals with respectively no TWA, with TWA and with TWA and T wave shifts in the different rows. The columns show the factor vectors for all tensor modes modes. Figures 8a, 8d and $8 \mathrm{~g}$ show the spatial vector for the signal without TWA, with TWA and with TWA and T wave shift respectively which show the sign and magnitude of the $\mathrm{T}$ wave in different channels. From Figure $8 \mathrm{a}$ we can for example conclude that $\mathrm{T}$ waves in channel 9 had a larger amplitude than those in channel 12. The temporal vectors, shown in Figure 8b, 8e and 8h correspond to the shape of the $\mathrm{T}$ wave in time. Here the difference between CPD and PARAFAC2 is obvious, especially in Figure $8 \mathrm{~h}$. The result for PARAFAC2 is a matrix, and each row of the matrix (corresponding to the temporal profile for each heartbeat) is plotted on top of each other. The presence of a large $\mathrm{T}$ wave shift in the third signal is evident. While CPD succeeded in capturing the mean shape of the $\mathrm{T}$ wave, the difference in the timing of the subsequent waves was discarded. This information is contained in the feature matrix obtained by PARAFAC2. The other signals did not contain a large shift, as is demonstrated by the small variation in the PARAFAC2 results of Figure 8b and 8e. Hence, the results of CPD and PARAFAC2 were much more similar.

TWA can be detected by analyzing the third loading vector. Figures $8 \mathrm{c}$, $8 \mathrm{f}$ and $8 \mathrm{i}$ show this loading vector for all analyzed signals. On Figure $8 \mathrm{c}$ we see that, although there is a certain variation in the $T$ wave magnitude over different heartbeats, no clear pattern is visible. The typical ABABAB-pattern is however clearly visible on Figure $8 \mathrm{f}$ and 8i. Note that in Figure 8f, there is only $\mathrm{T}$ wave alternans present in the first part of the signal. The results for PARAFAC2 and CPD are almost identical in Figure 8c and 8f, while the results in Figure $8 \mathrm{i}$ differ significantly. This is caused by the large $\mathrm{T}$ wave shift (see Figure 8i).

\section{MLSVD}

Apart from using CPD and PARAFAC2, TWA can also be detected using the MLSVD as main building block. Furthermore, since noise can seriously alter the ECG signal in the time domain, we first transform the signal to the multiscale wavelet domain using DWT to perform a more robust detection. The first aim is to extract the only $\mathrm{T}$-wave morphology from the 
ECG signal. Multiscale analysis, as discussed earlier, alleviates this problem by grossly segmenting the ECG morphological features where the signal of interest (T-wave with/without TWA) and noise are placed into different subbands. To get back the time-domain signal from the wavelet coefficients in a subband, these coefficients are synthesized in that subband, which is the same as applying inverse DWT to that subband. This process collectively is termed as multiscale analysis-by-synthesis (MAS). Since the objective is to extract the only T-wave morphology whose frequency is concentrated in between 0.3 and $15 \mathrm{~Hz}$ [38], synthesized signals of low-frequency $\left(A_{6}, D_{6}\right.$, and $D_{5}$ ) subbands are added (here, $L^{1}=6$ as the sampling frequency of the signal is $500 \mathrm{~Hz}$ ). The maximum frequency of these subbands are 3.906, $7.812,15.624 \mathrm{~Hz}$, respectively. Hence, the T-wave information is retained in these three subbands.

After obtaining $L+1$ subband tensors (ref, Section 4.1), the T-wave tensor is obtained as follows:

Reconstruct the subband tensors using multiscale synthesis on mode-2 fibers of $\mathcal{A}_{6}, \mathcal{D}_{6}$ and $\mathcal{D}_{5}$.

Add the reconstructed samples to get the T-wave tensor,

$$
\mathcal{R}=\mathbf{r}_{i: k}=\mathbf{a}_{6 i: k}+\mathbf{d}_{6 i: k}+\mathbf{d}_{5 i: k}, \quad i=1: I, k=1: K
$$

where $\mathbf{a}_{6 i: k}, \mathbf{d}_{6 i: k}$, and $\mathbf{d}_{5 i: k}$ are the reconstructed fibers. Similarly to [57] where PCA was applied to improve the TWA analysis performance by exploiting the spatial redundancy, here, MLSVD is employed on the T-wave tensor $\mathcal{R}$ for the same purpose by exploiting both spatial and temporal redundancy.

For comparison purpose of the MAS-MLSVD method over the multi-PCA (a matrix-based) method [57], we discuss here the results on a semi-synthetic database. It is created by adding different TWA amplitude levels ranging from 0 to $100 \mu \mathrm{Vs}$ to every other T-wave of ECG signals. The signals are considered from 52 records of healthy subjects' in the PTB database [69]. The addition of alternate waveforms is carried out with selected but randomly chosen leads rather than with all 8-leads. Further, to experience a high degree of realism, four types of noise, namely Gaussian, Laplacian, electrode motion, and muscular activity are considered and with different noise levels ranging from 0 to $50 \mathrm{~dB}$. Figure 9 shows the mean and standard deviation of the estimated alternans level for each input alternans level for these two methods, and also in the presence of laplacian noise of $20 \mathrm{~dB}$. Noise is added to verify the robustness of the proposed method. For input alternans levels ranging from 10 to $50 \mu \mathrm{V}$, difference between the mean estimated alternans level and input alternans level in case of multi-PCA method is significantly higher than the MAS-MLSVD method (for instance, 16.56 versus $0.48 \mu \mathrm{V}$ and 14.93 versus $0.07 \mu \mathrm{V}$ when input alternans levels are $20 \mu \mathrm{V}$ and $50 \mu \mathrm{V}$, respectively). In the presence of noise, the estimation accuracy of the multi-PCA degrades

\footnotetext{
${ }^{1} L=\left\lfloor\log _{2} F_{s}-2.96\right\rfloor=\left\lfloor\log _{2} 500-2.96\right\rfloor=6 .[4]$
} 


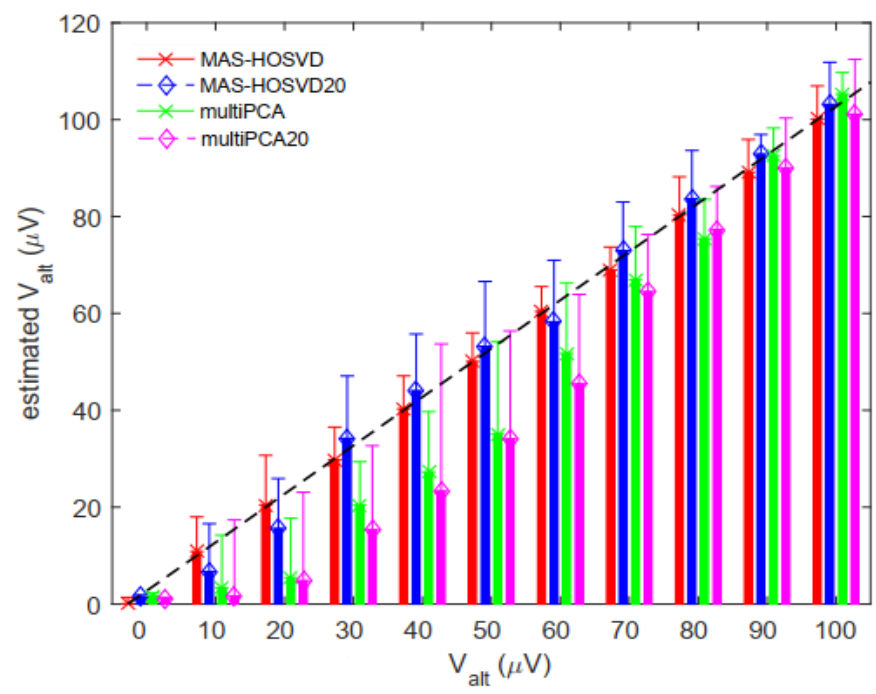

Fig. 9: Variation of estimated vs input alternans level in semi-synthetic datasets with $l p$ noise of SNR $=20 \mathrm{~dB}$ for the multi-PCA and MAS-MLSVD techniques. Markers and bars represent the mean and standard deviation of the estimated TWA amplitude, respectively. Dashed straight line represents the would-be (perfect) estimated alternans levels. Adapted from [67].

comparatively more than the MAS-MLSVD method. Additionally, for input alternans levels from 10 to $60 \mu \mathrm{V}$, the standard deviations observed are 15$30 \mu \mathrm{V}$. This indicates the inconsistency in estimation accuracy results of the multi-PCA scheme in the presence of noise.

To our knowledge, the Physionet TWA database is the only publicly accessible labeled TWA database. This makes it ideal for use as a benchmarking tool to make comparison with existing methods possible. It consists of 100 multilead ECG signals that are ranked based on their TWA level. Comparing different methods can be done by ranking the results from the TWA detection in order of magnitude, and comparing this ranking with the reference ranking by calculating the Kendall rank correlation coefficient between the two. The results for the three tensor-based approaches and some of the most wellknown methods from literature can be found in Table 3. It is clear that all tensor methods outperform the matrix-based alternatives. This could be expected, since it was already proven that combining information from all leads increased results for TWA detection [15]. The method that used PARAFAC2 obtains the best result overall while the results for CPD and MLSVD are comparable. Using a more general tensor decomposition method thus has clear added value in this application. The advantage of the MLSVD-based approach is however that it can also be used to assess TWA in each lead 
individually, and can thus be used to differentiate between different locations of TWA similarly to the MI detection method discussed earlier.

Table 3: Kendall coefficient scores obtained by comparing the rankings from different methods found in literature and the two proposed tensor-based methods with the reference ranking for the Physionet database. The tensorbased approaches reach the highest scores.

\begin{tabular}{lc}
\hline Method & Kendall coefficient \\
\hline CPD & 0.79 \\
PARAFAC2 & 0.87 \\
MLSVD & 0.81 \\
Periodic Component Analysis [57] & 0.77 \\
Modified Moving Average Method [61] & 0.73 \\
Spectral Method [78] & 0.42 \\
\hline
\end{tabular}

\subsection{Analysis of changes in heartbeat morphology}

The final application discussed in this chapter is the analysis of changes in heartbeat morphology prior to cardiorespiratory arrest in the intensive care unit, explained in $[33,35]$. For this study, a dataset with long-term patients in the intensive care unit was collected. The data were however characterized by large amounts of noise, diminishing the signal quality and possibly affecting the analysis results. While the corrupted portions could be removed prior to the analysis, this seriously reduces the amount of information available. Furthermore, since often not all leads are contaminated by noise, removing the corrupted portions also eliminates clean ECG signal that contains valuable information. The method proposed here introduces the use of weighted tensor decompositions, which allow us to incorporate prior knowledge about the signal quality in the tensor decomposition in the form of a weight tensor [62]. When choosing the weights properly, they can automatically deal with the noise that is inherent to biomedical signals, leading to a more accurate analysis and making them better suited to work with real-life signals. The method used in this study furthermore uses a computationally efficient weighting scheme [12], which is essential for real-time processing.

As explained before, CPD writes a tensor $\mathcal{T}$ as a sum of rank-one terms. Here, we used an alternative way to compute the CPD, namely Weighted CPD (WCPD) which uses weighted least-squares instead of regular leastsquares. WCPD permits the incorporation of prior knowledge about the sig- 
nal quality in the tensor decomposition, giving lower weight to entries with higher noise levels. This is done by introducing a weight tensor $\mathcal{W}$ with the same dimensions as $\mathcal{T}$ into the standard CPD optimization problem. Each entry of $\mathcal{W}$ contains the weight for the corresponding entry of $\mathcal{T}$. A detailed explanation of the weight tensor construction follows in the next paragraph. The new optimization problem is then:

$$
\min _{\mathbf{A}, \mathbf{B}, \mathbf{C}} \frac{1}{2}\|\mathcal{W} *(\mathcal{T}-[\mathbf{A}, \mathbf{B}, \mathbf{C}])\|_{F}^{2}
$$

The optimization problem can be solved solved using a novel Weighted Least Squares (WLS) approach where the weight tensor is modeled by a polyadic decomposition, enabling efficient weighting. The computational details of the WLS algorithm can be found in [12].

The weight tensor $\mathcal{W}$ contains information about the signal quality, e.g. entries with higher quality receive higher weights. The quality of ECG signals is reduced by artifacts, which are technical or physiological. Technical artifacts can be caused by equipment malfunctioning or electrode loosening. During a technical artifact no ECG signal is measured; the corresponding entries in $\mathcal{T}$ therefore receive a weight of 0 , effectively eliminating them from further analysis. Physiological artifacts are caused by for example muscle contractions and are superimposed onto the ECG signal, reducing the Signal-to-Noise Ratio (SNR). For signals which do not contain technical artifacts an estimate of the SNR was therefore used as weight. We calculated one weight for each complete mode-2 fiber, e.g. each full heartbeat in each channel. It was thus assumed that the signal quality was the same during the time course of one heartbeat but could differ from channel to channel or between different heartbeats. Hence, the resulting weight tensor $\mathcal{W}$ has rank $M$, by construction, with $M$ equal to the number of channels in the ECG signal.

The result of the WCPD with rank 1 are three factor vectors that are identical to the ones shown in Figure 7. For this application, the second factor vector, which contains the temporal profile of the main heartbeat in the signal, is analyzed and processed further. Standard techniques [48] can be used to detect fiducial points on the $\mathrm{P}$ wave, QRS complex and $\mathrm{T}$ wave from which interval- and amplitude-parameters can be derived. By applying the tensor decomposition with a sliding window of 100 heartbeats, we could track and analyze the changes of these parameters in time.

From these analyses, explained in more detail in [33, 35], it could be concluded that changes in interval lengths are more significant between groups of patients with different causes of cardiac arrest than changes in amplitude. Changes in amplitude, especially for $\mathrm{P}$ wave and $\mathrm{T}$ wave amplitude, are however more prevalent overall and might thus be useful to monitor overall patient deterioration. To confirm this, it would be beneficial to collect data from a group of control patients that do not experience a code blue to verify the normal physiological variations in different parameters. 
The Weighted Least-Squares approach used to compute the WCPD optimization problem is computationally much more efficient than previous weighted tensor decomposition methods. This is extremely beneficial for use in continuous patient monitoring, where the delay between the physiological change and the algorithmic output should be minimal. The current method gives an output for each sliding window of 100 heartbeats (1-1.5 minutes), resulting in a quasi-real time monitoring of ECG morphology. Recent advances in tensor methodology however focus on the development of efficient tensor updating methods [83]. This class of methods calculate a new tensor decomposition every time new data arrives (for example after every heartbeat) in a time- and memory-efficient way. Their use would lead to an improvement in the time resolution of the results which could help in gaining a deeper understanding in the timing of the changes in ECG morphology before cardiac arrest.

\section{Conclusions and Future directions}

Tensors are a novel concept in ECG signal processing. This chapter gave an overview of different applications where tensors have been used to solve problems in cardiac processing. As such we have shown that tensor-based methods are very suitable to represent data with multiple modes, and lead to improved results compared to matrix-based methods when multiple leads need to be processed simultaneously.

We gave an overview of five typical ECG applications: data compression, myocardial infarction detection and localization, irregular heartbeat classification, TWA detection and quantification, and analysis of changes in heartbeat morphology. Most of the methods presented are however highly flexible and can be used in different applications with minimal changes. This can be done by either including different segments of the ECG signal in the tensor or by extracting different features from the decomposition results.

The popularity of tensor-based algorithms has increased in the past years and it is expected that this increase will continue in the near future. We can, therefore, define possible future directions for the use of tensors in ECG applications.

The use of tensor decompositions for single lead ECG signal is a first future direction that has a lot of potential. As more and more wearable devices are equipped with ECG recording devices, the amount of single lead ECG signals is expected to increase drastically in the next years. Single lead ECG signals require an extra tensorization step prior to tensor decomposition methods, but since many tensorization techniques have been developed [22], this should not be a large bottleneck. The use of tensor updating was briefly explored in the previous section. It is a promising approach for most real-time monitoring methods, where an immediate output is required. Here, tensor updating 
was applied in a very straightforward way, by updating the result of the tensor decomposition for each new heartbeat. The updating could, however, be taken even further, by for example not only updating the results of the tensor decomposition but also updating the rank of the decomposition as the signals are changing over the course of time. Tensors can also be applied for data fusion, where signals from different sources are analyzed simultaneously. While joint analysis of measurements from different signal types has not been explored in this chapter, it has the potential to reveal additional features that describe the interaction between different biosignals. It is for example known that the cardiorespiratory coupling, which describes the influences of respiration on heart rate and blood pressure, is different for healthy and sick people. Using data fusion approaches on the combination of ECG and respiration or blood pressure would open the door to the development of algorithms to analyze a whole new class of biomarkers.

Acknowledgments This research was supported by: imec funds 2017, Belgian Foreign Affairs Development Cooperation: VLIR-UOS programs (20132019), Fonds de la Recherche Scientifique - FNRS and Fonds Wetenschappelijk Onderzoek - Vlaanderen under EOS Project no 30468160 (SeLMA), Research Council KU Leuven: C1 project C16/15/059-nD, European Research Council: The research leading to these results has received funding from the European Research Council under the European Union's Seventh Framework Programme (FP7/2007-2013) / ERC Advanced Grant: BIOTENSORS (no. 339804). This paper reflects only the authors' views and the Union is not liable for any use that may be made of the contained information. Griet Goovaerts is a SB Ph.D. fellow at Fonds voor Wetenschappelijk Onderzoek (FWO), Vlaanderen, supported by the Flemish government.

\section{References}

1. Abenstein JP, Tompkins WJ (1982) A new data-reduction algorithm for real-time ECG analysis. IEEE Trans. on Biomed. Eng. BME-29(1):43-48.

2. Ansari S, Farzaneh N, Duda M, Horan K, Andersson HB, Goldberger ZD, Nallamothu BK, Najarian K (2017) A Review of Automated Methods for Detection of Myocardial Ischemia and Infarction Using Electrocardiogram and Electronic Health Records. IEEE Reviews in Biomedical Engineering 10:264-298.

3. Akbari H, Shamsollahi MB, Phlypo R (2015) Fetal ecg extraction using $\pi$ tucker decomposition. In:2015 International Conference on Systems, Signals and Image Processing (IWSSIP), Sept 2015, p 174-178.

4. Al-Fahoum AS (2006) Quality assessment of ECG compression techniques using a wavelet-baseddiagnostic measure. IEEE Transactions on Information Technology in Biomedicine 10(1):182-191.

5. Arif M, Malagore I, Afsar F (2012) Detection and localization of myocardial infarction using k-nearest neighbor classifier. J. of Med. Syst. 36(1):279-289.

6. Bergqvist G, Larsson EG (2010) The higher-order singular value decomposition: Theory and an application. IEEE Sig. Process. Mag. 27(3):151-154. 
7. Bharath HN, Sauwen N, Sima DM, Himmelreich U, De Lathauwer L, Van Huffel S (2016) Canonical polyadic decomposition for tissue type differentiation using multi-parametric MRI in high-grade gliomas. In: 24th European Signal Processing Conference (EUSIPCO), Budapest, 2016, p 547-551.

8. Bharath HN, Sima DM, Sauwen N, Himmelreich U, De Lathauwer L, Van Huffel S (2017) Non-Negative Canonical Polyadic Decomposition for Tissue Type Differentiation in Gliomas. IEEE Journal of Biomedical and Health Informatics 21(4):11241132.

9. Bortolan G, Christov II (2008) Principal component analysis for detection and assessment of T-wave alternans. In: Computers in Cardiology, Sept 2008, p 521524 .

10. Boussé M, Goovaerts G, Vervliet N, Debals O, Van Huffel S, De Lathauwer L (2017) Irregular heartbeat classification using Kronecker product equations. In: 39th Annual International Conference of the IEEE Engineering in Medicine and Biology Society (EMBC 2017), July 2017, p 438-441.

11. Boussé M, Vervliet N, Domanov I, Debals O, De Lathauwer L (2018) Linear Systems with a Canonical Polyadic Decomposition Constrained Solution: Algorithms and Applications. Numer Linear Algebra Appl. 2018:e2190.

12. Boussé M. De Lathauwer L (2017) Nonlinear Least Squares Algorithm for Canonical Polyadic Decomposition Using Low-Rank Weights. In: 7th International Workshop on Computational Advances in Multi-Sensor Adaptive Processing. Curaçao, Dutch Antilles, 2017, p 39-43.

13. Bro R, Andersson C, Kiers H (1999) Parafac2 - part ii. modeling chromatographic data with retention time shifts. Journal of Chemometrics 309:295--309.

14. Bro R, Kiers HAL (2003) A new efficient method for determining the number of components in PARAFAC models. Journal of Chemometrics 17:274--286.

15. Burattini L, Man S, Burattini R, Swenne CA (2012) Comparison of standard versus orthogonal ecg leads for t-wave alternans identification. Annals of Noninvasive Electrocardiology 17(2):130-140.

16. Cetin AE, Koymen H, Aydin MC (1993) Multichannel ECG data compression by multirate signal processing and transform domain coding techniques. IEEE Trans. on Biomed. Eng. 40(5):495-499.

17. Cichocki A, Mandic DP, De Lathauwer L, Zhou G, Zhao Q, Caiafa CF, Phan AH (2015) Tensor decompositions for signal processing applications: From two-way to multiway component analysis. IEEE Signal Processing Magazine 32(2):145-163.

18. Cong F, Nandi AK, He Z, Cichocki A, Ristaniemi T (2012) Fast and effective model order selection to determine the number of sources in a linear transformation model. In: Proceedings of the 20th European Signal Processing Conference (EUSIPCO). Bucharest, Romania, 2012, p 1870--1874.

19. Cox JR, Nolle FM, Fozzard HA, Oliver GC (1968) AZTEC: A preprocessing scheme for real-time ECG rhythm analysis. IEEE Trans. on Biomed. Eng. BME-15:128129.

20. Dauwels J, Srinivasan K, Reddy MR, Cichocki A (2013) Near-lossless multichannel EEG compression based on matrix and tensor decompositions. IEEE J. of Biomed. and Health Info. 17(3):708-714.

21. Debals O, De Lathauwer L (2015) Stochastic and Deterministic Tensorization for Blind Signal Separation. Latent Variable Analysis and Signal Separation, p 3--13.

22. Debals O, De Lathauwer L (2017) The concept of tensorization. Technical Report 17-99, ESAT-STADIUS, KU Leuven (Leuven, Belgium).

23. De Lathauwer L, De Moor B, Vandewalle J (2000) A multilinear singular value decomposition. SIAM Journal on Matrix Analysis and Applications 21(4):12531278 .

24. De Lathauwer L, Vandewalle J (2004) Dimensionality reduction in higher-order signal processing and rank- $\left(R_{1}, R_{2}, \cdots, R_{N}\right)$ reduction in multilinear algebra. Linear Algebra and its Applications 391:31-55. 
25. Domanov I, De Lathauwer L (2013) On the uniqueness of the canonical polyadic decomposition of third-order tensors - Part II: Uniqueness of the overall decomposition. SIAM Journal on Matrix Analysis and Applications 34(3):876-903.

26. Domanov I, De Lathauwer L (2015) Generic uniqueness conditions for the canonical polyadic decomposition and INDSCAL. SIAM Journal on Matrix Analysis and Applications 36(4):1567-1589.

27. Domanov I, De Lathauwer L (2017) Canonical polyadic decomposition of thirdorder tensors: Relaxed uniqueness conditions and algebraic algorithm. Linear Algebra and its Applications 513:342-375.

28. Geirnaert S, Goovaerts G, Padhy S, Boussé M, De Lathauwer L, Van Huffel S (2018) Tensor-based ECG signal processing applied to atrial fibrillation detection. In: Proceedings of the 52th IEEE Asilomar conference, Pacific Grove, CA, USA, 2018.

29. Goovaerts G, Varon C, Vandenberk B, Willems R, Van Huffel S (2014) Tensorbased detection of $\mathrm{T}$ wave alternans in multilead ECG signals. In: Computing in Cardiology Conference, Sept 2014, p 185-188.

30. Goovaerts G, De Wel O, Vandenberk B, Willems R, Van Huffel S (2015) Detection of Irregular Heartbeats using tensors. In: 42nd annual conference of computing in cardiology, Sept 2015.

31. Goovaerts G, Vandenberk B, Willems R, Van Huffel S (2015) Tensor-based detection of $\mathrm{T}$ wave alternans using ECG. In: Proc. of the 37th Annual International Conference of the IEEE Engineering in Medicine and Biology Society of the IEEE (EMBC), Milan, Italy, Aug. 2015, p 6991-6994.

32. Goovaerts G, Vandenberk B, Willems R, Van Huffel S (2017) Automatic detection of $\mathrm{T}$ wave alternans using tensor decompositions in multilead ECG signals. Physiol. Meas. 38(8):1513-1528.

33. Goovaerts G, Van Huffel S, Hu X (2017) Tensor-based Analysis of ECG changes prior to in-hospital cardiac arrest. In: Proc. of the 44rd Annual Computing in Cardiology (CinC 2017), CinC, Rennes, France, Sep. 2017.

34. Goovaerts G, Willems R, Van Huffel S. (2018) Tensor-based ECG analysis in Sudden Cardiac Death. PhD thesis, Department of Electrical Engineering, KU Leuven. December 2018.

35. Goovaerts G., Boussé M, Do D., De Lathauwer L., Van Huffel S., Hu X. Analysis of changes in ECG morphology prior to in-hospital cardiac arrest using weighted tensor decompositions. submitted for publication.

36. Håstad J (1990) Tensor rank is NP-complete. J. Algorithms, 11(4):644-654.

37. Hayn D, Kollmann A, Schreier G (2006) Automated QT interval measurement from multilead ECG signals. Computing in Cardiology, Valencia, Spain, 2006, p 381--384.

38. Hearing BD, Stone PH, Verrier RL (1996) Frequency response characteristics required for detection of T-wave alternans during ambulatory ECG monitoring. Ann. Noninvas. Electrocardiol. 1(2):103-112.

39. Hillar CJ, Lim LH (2009) Most Tensor Problems are NP-Hard. Tech. Rep., Eprint arXiv:0911.1393v4

40. Huang K, Zhang L (2014) Cardiology knowledge free ECG feature extraction using generalized tensor rank one discriminant analysis. EURASIP Journal on Advances in Signal Processing 2014(1).

41. Ikeda T, Saito H, Tanno K, Shimizu H, Watanabe J, Ohnishi Y, Kasamaki Y, Ozawa Y (2002) T-Wave Alternans as a Predictor for Sudden Cardiac after Myocardial Infarction. The American Journal of Cardiology 89(1):79-82.

42. Ishteva M, Absil PA, Van Huffel S, De Lathauwer L (2011) Best Low Multilinear Rank Approximation of Higher-Order Tensors, Based on the Riemannian TrustRegion Scheme. SIAM Journal on Matrix Analysis and Applications 32(1):115--135.

43. Jalaleddine S, Hutchens C, Stratran R, Coberly W (1990) ECG data compression techniques - a unified approach. IEEE Trans. on Biomed. Eng. 37(4):329-343. 
44. Jayachandran E, Joseph KP, Acharya UR (2010)Analysis of myocardial infarction using discrete wavelet transform. J. of Med. Syst. 34(6):985-992.

45. Kolda TG, Bader BW (2009) Tensor decompositions and applications. SIAM REVIEW 51(3):455-500.

46. Kuang L, Hao F, Yang L, Lin M, Luo C, Min G (2014) A tensor-based approach for big data representation and dimensionality reduction. IEEE Trans. on Emerging Topics in Computing 2(3):280-291.

47. Laguna P, Jane R, Caminal P (1994) Automatic Detection of Wave Boundaries in Multilead ECG Signals: Validation with the CSE Database. Computers and Biomedical Research 27:45--60.

48. Laguna P, Martinez Cortes JP, Pueyo E (2016) Techniques for Ventricular Repolarization Instability Assessment From the ECG. Proceedings of the IEEE 104(2):392415.

49. León AAS, Goovaerts G, Seisdedos CRV, Van Huffel S (2016) Irregular heartbeats detection using tensors and support vector machines. In: 2016 Computing in Cardiology Conference, Sept 2016, p 1037-1040.

50. Lee H, Buckley KM (1999) ECG data compression using cut and align beats approach and 2-D transforms. IEEE Trans. on Biomed. Eng. 46(5):556-564.

51. Li Q, Shi X, Schonfeld D (2013) Robust HOSVD-based higher-order data indexing and retrieval. IEEE Signal Processing Letters 20(10):984-987.

52. Llamedo M, Khawaja A, Martínez J (2010) Analysis of 12-lead classification models for ECG classification. In: Computing in Cardiology, Sept. 2010, p 673--676.

53. Lu HL, Ong K, Chia P (2000) An automated ECG classification system based on a neuro-fuzzy system. In: Computers in Cardiology, 2000, p 387-390.

54. Martinez J, Olmos S (2005) Methodological principles of T wave alternans analysis: a unified framework. IEEE Trans. on Biomed. Eng. 52(4):599-613.

55. Manikandan MS, Dandapat S (2007) Wavelet energy based diagnostic distortion measure for ECG. Biomedical Signal Processing and Control, Elsevier 2:80-96.

56. Manikandan MS, Dandapat S (2008) Wavelet threshold based TDL and TDR algorithms for real-time ECG signal compression. Biomedical Signal Processing and Control, Elsevier 3:44-66.

57. Monasterio V, Laguna P, Martinez J (2009) Multilead analysis of T-wave alternans in the ECG using principal component analysis. IEEE Trans. on Biomed. Eng. 56(7):1880-1890.

58. Monasterio V, Clifford GD, Laguna P, Martínez JP (2010) A multilead scheme based on periodic component analysis for T-wave alternans analysis in the ECG. Annals of Biomed. Eng. 38(8):2532-2541.

59. Mueller W (1978) Arrhythmia detection program for an ambulatory ECG monitor. Biomedical sciences instrumentation 14:81-85.

60. Narayan SM, Smith JM (1999) Spectral analysis of periodic fluctuations in electrocardiographic repolarization. IEEE Trans. on Biomed. Eng. 46(2):203-212.

61. Nearing BD, Verrier RL (2002) Modified moving average analysis of T-wave alternans to predict ventricular fibrillation with high accuracy. Journal of applied physiology (Bethesda, Md. : 1985) 92(2):541--549.

62. Paatero P (1997) A weighted non-negative least squares algorithm for three-way 'PARAFAC' factor analysis. Chemometrics and Intelligent Laboratory Systems 38(2):223--242.

63. Padhy S, Dandapat S (2015) Exploiting multi-lead electrocardiogram correlations using robust third-order tensor decomposition. Healthcare Technology Letters. 2(5):112-117.

64. Padhy S, Sharma L, Dandapat S (2016) Multilead ECG data compression using SVD in multiresolution domain. Biomedical Signal Processing and Control, Elsevier $23: 10-18$. 
65. Padhy S, Dandapat S (2017) Third-order tensor based analysis of multilead ECG for classification of myocardial infarction, Biomedical Signal Processing and Control, Elsevier 31:71-78.

66. Padhy S, Multilead ECG Data Analysis using SVD and Higher-Order SVD (2017) In:PhD Thesis, Indian Institute of Technology Guwahati, India, March 2017. [Online]. Available: http://gyan.iitg.ernet.in/handle/123456789/1006

67. Padhy S, Dandapat S (2018) Validation of $\mu$-volt T-Wave Alternans Analysis using Multiscale Analysis-by-Synthesis and Higher-Order SVD, Biomedical Signal Processing and Control, Elsevier 40:171-179.

68. Pan J, Tompkins WJ (1985) A real-time qrs detection algorithm.IEEE Trans. on Biomed. Eng. BME-32(3):230-236.

69. Goldberger AL, et al. (2000), Physiobank, physiotoolkit, and physionet: Components of a new research resource for complex physiologic signals. circulation 101(23):e215-e220 (June 13, 2000).

70. Ramakrishnan AG, Saha S (1997)ECG coding by wavelet-based linear prediction. IEEE Trans. on Biomed. Eng. 44(12):1253-1261.

71. Reddy MRS, Edenbrandt L, Svensson J, Haisty WK, Pahlm O (1992) Neural network versus electrocardiographer and conventional computer criteria in diagnosing anterior infarct from the ECG. In:Computers in Cardiology, Oct 1992, p 667-670.

72. Ribeiro LN, Hidalgo-Muñoz AR, Favier G, Mota JCM, De Almeida ALF, Zarzoso V (2015) A tensor decomposition approach to noninvasive atrial activity extraction in atrial fibrillation ecg. In:23rd European Signal Processing Conference (EUSIPCO2015), Aug 2015, p 2576-2580.

73. Sharma LN, Dandapat S, Mahanta A (2012) Multichannel ECG data compression based on multiscale principal component analysis. IEEE Trans. on Info. Tech. in Biomed. 16(4):730-736.

74. Sharma LN, Tripathy R, Dandapat S (2015) Multiscale energy and eigenspace approach to detection and localization of myocardial infarction. IEEE Trans. on Biomed. Eng. 62(7):1827-1837.

75. Sidiropoulos N, De Lathauwer L, Fu X, Huang K, Papalexakis E, Faloutsos C (2017) Tensor decomposition for signal processing and machine learning. IEEE Transactions on Signal Processing, 65(13):3551-3582.

76. Singh B, Kaur A, Singh J (2015) A Review of ECG Data Compression Techniques, International Journal of Computer Applications, 116(11):39-44.

77. Simoliuniene R, Krisciukaitis A, Macas A, Baksyte G, Saferis V, Zaliunas R (2008) Principal component analysis based method for detection and evaluation of ECG T-wave alternans. In: Computers in Cardiology, Sept 2008, p 757-760.

78. Smith JM, Clancy EA, Valeri CR, Ruskin JN, Cohen RJ (1988) Electrical alternans and cardiac electrical instability. Circulation 77(1):110-121.

79. Srinivasan K, Dauwels J, Reddy MR (2013) Multichannel EEG compression: Wavelet-based image and volumetric coding approach. IEEE Journal of Biomed. and Health Info. 17(1):113-120.

80. Sun L, Lu Y, Yang K, Li S (2012) ECG analysis using multiple instance learning for myocardial infarction detection. IEEE Trans. on Biomed. Eng. 59(12):3348-3356.

81. Vervliet N, Debals O, De Lathauwer L (2016) Tensorlab 3.0 - Numerical optimization strategies for large-scale constrained and coupled matrix/tensor factorization. In: 50th Asilomar Conference on Signals, Systems and Computers. IEEE, 2016, p $1733-1738$.

82. Thygesen K, Alpert JS, Jaffe AS, Simoons ML, Chaitman BR, White HD (2012) Third universal definition of myocardial infarction. Circulation 126(16):2020-2035.

83. Vandecappelle M, Boussé M, Vervliet N, De Lathauwer L (2017) CPD Updating using Low-Rank Weights. In: 25th European Signal Processing Conference (EUSIPCO). Kos, Greece, 2017, p 663--557. 
84. Van Eyndhoven S, Boussé M, Hunyadi B, De Lathauwer L, Van Huffel S (2018) Single-channel EEG classification by multi-channel tensor subspace learning and regression. In: Proc. of the 28th IEEE International Workshop on Machine Learning for Signal Processing (MLSP 2018), September 2018.

85. Vasilescu M, Terzopoulos D (2007) Multilinear (tensor) image synthesis, analysis, and recognition [exploratory dsp]. IEEE Sig. Process. Mag. 24(6):118-123.

86. Vervliet N, Debals O, Sorber L, De Lathauwer L (2014) Breaking the curse of dimensionality using decompositions of incomplete tensors: Tensor-based scientific computing in big data analysis. IEEE Signal Processing Magazine 31(5):71-79.

87. Vítek M, Hrubeš J, Kozumplík J (2009) A Wavelet-Based ECG Delineation in Multilead ECG Signals: Evaluation on the CSE Database. World Congress on Medical Physics and Biomedical Engineering. Springer, Berlin, Heidelberg, 2009, p $177-$ 180.

88. Wei J, Chang C, Chou N, Jan G (2001) ECG data compression using truncated singular value decomposition. IEEE Trans. on Info. Tech. in Biomedicine 5(4):290299. 